MIT-CTP-3689

HUTP-05/A0044

\title{
The Minimal Model for Dark Matter and Unification
}

\author{
Rakhi Mahbubani $^{a}$, Leonardo Senatore $^{b, *}$ \\ a Jefferson Physical Laboratory, \\ Harvard University, Cambridge MA 02138 \\ ${ }^{b}$ Center for Theoretical Physics, \\ Massachusetts Institute of Technology, Cambridge, MA 02139
}

\begin{abstract}
Gauge coupling unification and the success of $\mathrm{TeV}$-scale weakly interacting dark matter are usually taken as evidence of low energy supersymmetry (SUSY). However, if we assume that the tuning of the higgs can be explained in some unnatural way, from environmental considerations for example, SUSY is no longer a necessary component of any Beyond the Standard Model theory. In this paper we study the minimal model with a dark matter candidate and gauge coupling unification. This consists of the SM plus fermions with the quantum numbers of SUSY higgsinos, and a singlet. It predicts thermal dark matter with a mass that can range from $100 \mathrm{GeV}$ to around $2 \mathrm{TeV}$ and generically gives rise to an electric dipole moment (EDM) that is just beyond current experimental limits, with a large portion of its allowed parameter space accessible to next generation EDM and direct detection experiments. We study precision unification in this model by embedding it in a 5-D orbifold GUT where certain large threshold corrections are calculable, achieving gauge coupling and $b-\tau$ unification, and predicting a rate of proton decay just beyond current limits.
\end{abstract}

\section{Introduction}

Over the last few decades the search for physics beyond the Standard Model (SM) has largely been driven by the principle of naturalness, according to which the parameters of a low energy effective field theory like the SM should not be much smaller than the contributions that come from running them up to the cutoff. This principle can be used to constrain the couplings of the effective theory with positive mass dimension, which have a strong dependence on UV physics. Requiring no fine tuning between bare parameters and the corrections they receive from renormalization means that the theory must have a low cutoff. New physics can enter at this scale to literally cut off the high-energy contributions from renormalization.

${ }^{*}$ This work is supported in part by funds provided by the U.S. Department of Energy (D.O.E) under cooperative research agreement DF-FC02-94ER40818 
In the specific case of the SM the effective lagrangian contains two relevant parameters: the higgs mass and the cosmological constant (c.c), both of which give rise to problems concerning the interpretation of the low energy theory. Any discussion of large discrepancies between expectation and observation must begin with what is known as the c.c. problem. This relates to our failure to find a well-motivated dynamical explanation for the factor of $10^{120}$ between the observed c.c and the naive contribution to it from renormalization which is proportional to $\Lambda^{4}$, where $\Lambda$ is the cutoff of the theory, usually taken to be equal to the Planck scale. Until very recently there was still hope in the high energy physics community that the c.c. might be set equal to zero by some mysterious symmetry of quantum gravity. This possibility has become increasingly unlikely with time since the observation that our universe is accelerating strongly suggests the presence of a non-zero cosmological constant [1, 2].

A less extreme example is the hierarchy between the higgs mass and the GUT scale which can be explained by SUSY breaking at around a TeV. Unfortunately the failure of indirect searches to find light SUSY partners has brought this possibility into question, since it implies the presence of some small fine-tuning in the SUSY sector. This 'little hierarchy' problem [3, 4, raises some doubts about the plausibility of low energy SUSY as an explanation for the smallness of the higgs mass.

Both these problems can be understood from a different perspective: the fact that the c.c. and the higgs mass are relevant parameters means that they dominate low energy physics, allowing them to determine very gross properties of the effective theory. We might therefore be able to put limits on them by requiring that this theory satisfy the environmental conditions necessary for the universe not to be empty. This approach was first used by Weinberg [5] to deduce an upper bound on the cosmological constant from structure formation, and was later employed to solve the hierarchy problem in an analogous way by invoking the atomic principle [6].

Potential motivation for this class of argument can be found in the string theory landscape. At low energies some regions of the landscape can be thought of as a field theory with many vacua, each having different physical properties. It is possible to imagine that all these vacua might have been equally populated in the early universe, but observers can evolve only in the few where the low energy conditions are conducive to life. The number of vacua with this property can be such a small proportion of the total as to dwarf even the tuning involved in the c.c. problem; resolving the hierarchy problem similarly needs no further assumptions. This mechanism for dealing with both issues simultaneously by scanning all relevant parameters of the low energy theory within a landscape was recently proposed in [7, 8].

From this point of view there seems to be no fundamental inconsistency with having the SM be the complete theory of our world up to the Planck scale; nevertheless this scenario presents various problems. Firstly there is increasing evidence for dark matter (DM) in the universe, and current cosmological observations fit well with the presence of a stable weakly interacting particle at around the TeV scale. The SM contains no such particle. Secondly, from a more aesthetic viewpoint gauge couplings do not quite unify at high energies in the SM alone; adding weakly interacting particles changes the running so unification works better. A well-motivated example of a model that does this is Split Supersymmetry [7, which is however not the simplest possible theory of this type. In light of this we study the minimal model with a finely-tuned higgs and a good thermal dark matter candidate proposed in [8], which also allows for gauge coupling unification. Although a systematic analysis of the complete set of such models was carried out in [9], the simplest one we study here was missed because the authors did not consider the possibility of having large UV threshold corrections that fix unification, as well as a GUT mechanism suppressing proton decay.

Adding just two 'higgsino' doublets ${ }^{1}$ to the SM improves unification significantly. This model

\footnotetext{
${ }^{1}$ Here 'higgsino' is just a mnemonic for their quantum numbers, as these particles have nothing to do with
} 
is highly constrained since it contains only one new parameter, a Dirac mass term for the doublets (' $\mu$ '), the neutral components of which make ideal DM candidates for $990 \mathrm{GeV} \lesssim \mu \lesssim 1150 \mathrm{GeV}$ (see [9] for details). However a model with pure higgsino dark matter is excluded by direct detection experiments since the degenerate neutralinos have unsuppressed vector-like couplings to the $Z$ boson, giving rise to a spin-independent direct detection cross-section that is 2-3 orders of magnitude above current $\operatorname{limits}^{2}$ [10, 11]. To circumvent this problem, it suffices to include a singlet ('bino') at some relatively high energy $\left(\lesssim 10^{9} \mathrm{GeV}\right)$, with yukawa couplings with the higgsinos and higgs, to lift the mass degeneracy between the 'LSP' and 'NLSP'3 by order $100 \mathrm{keV}$ [12, as explained in Appendix A. The instability of such a large mass splitting between the higgsinos and bino to radiative corrections, which tend to make the higgsinos as heavy as the bino, leads us to consider these masses to be separated by at most two orders of magnitude, which is technically natural. We will see that the yukawa interactions allow the DM candidate to be as heavy as $2.2 \mathrm{TeV}$. There is also a single reparametrization invariant $\mathrm{CP}$ violating phase which gives rise to a two-loop contribution to the electron EDM that is well within the reach of next-generation experiments.

Our paper is organized as follows: in Section 2 we briefly introduce the model, in Section 3 we study the DM relic density in different regions of our parameter space with a view to constraining these parameters; we look more closely at the experimental implications of this model in the context of dark matter direct detection and EDM experiments in Sections 4 and 5. Next we study gauge coupling unification at two loops. We find that this is consistent modulo unknown UV threshold corrections, however the unification scale is too low to embed this model in a simple 4D GUT. This is not necessarily a disadvantage since 4D GUTs have problems of their own, in splitting the higgs doublet and triplet for example. A particularly appealing way to solve all these problems is by embedding our model in a 5D orbifold GUT, in which we can calculate all large threshold corrections and achieve unification. We also find a particular model with $b-\tau$ unification and a proton lifetime just above current bounds. We conclude in Section 7

\section{The Model}

As mentioned above, the model we study consists of the SM with the addition of two fermion doublets with the quantum numbers of SUSY higgsinos, plus a singlet bino, with the following renormalizable interaction terms:

$$
\mu \Psi_{u} \Psi_{d}+\frac{1}{2} M_{1} \Psi_{s} \Psi_{s}+\lambda_{u} \Psi_{u} h \Psi_{s}+\lambda_{d} \Psi_{d} h^{\dagger} \Psi_{s}
$$

where $\Psi_{s}$ is the bino, $\Psi_{u, d}$ are the higgsinos, $h$ is the finely-tuned higgs.

We forbid all other renormalizable couplings to SM fields by imposing a parity symmetry under which our additional particles are odd whereas all SM fields are even. As in SUSY conservation of this parity symmetry implies that our LSP is stable.

The size of the yukawa couplings between the new fermions and the higgs are limited by requiring

perturbativity to the cutoff. For equal yukawas this constrains $\lambda_{u}\left(M_{Z}\right)=\lambda_{d}\left(M_{Z}\right) \leq 0.88$, while if we take one of the couplings to be small, say $\lambda_{d}\left(M_{Z}\right)=0.1$ then $\lambda_{u}\left(M_{Z}\right)$ can be as large as 1.38.

The above couplings allow for the $\mathrm{CP}$ violating phase $\theta=\operatorname{Arg}\left(\mu M_{1} \lambda_{u}^{*} \lambda_{d}^{*}\right)$, giving 5 free parameters in total. In spite of its similarity to the MSSM (and Split SUSY) weak-ino sector, there

the SUSY partners of the higgs.

${ }^{2} \mathrm{~A}$ model obtained adding a single higgsino doublet, although more minimal, is anomalous and hence is not considered here.

${ }^{3}$ From here on we will refer to these particles and couplings by their SUSY equivalents without the quotation marks for simplicity. 
are a number of important differences which have a qualitative effect on the phenomenology of the model, especially from the perspective of the relic density. Firstly a bino-like LSP, which usually mixes with the wino, will generically annihilate less effectively in this model since the wino is absent. Secondly the new yukawa couplings are free parameters so they can get much larger than in Split SUSY, where the usual relation to gauge couplings is imposed at the high SUSY breaking scale. This will play a crucial role in the relic density calculation since larger yukawas means greater mixing in the neutralino sector as well as more efficient annihilation, especially for the bino which is a gauge singlet.

Our $3 \times 3$ neutralino mass matrix is shown below:

$$
M_{N}=\left(\begin{array}{ccc}
M_{1} & \lambda_{u} v & \lambda_{d} v \\
\lambda_{u} v & 0 & -\mu e^{i \theta} \\
\lambda_{d} v & -\mu e^{i \theta} & 0
\end{array}\right)
$$

for $v=174 \mathrm{GeV}$, where we have chosen to put the $\mathrm{CP}$ violating phase in the $\mu$ term. The chargino is the charged component of the higgsino with tree level mass $\mu$.

It is possible to get a feel for the behavior of this matrix by diagonalizing it perturbatively for small off-diagonal terms, this is done in Appendix A.

\section{Relic Abundance}

In this section we study the regions of parameter space in which the DM abundance is in accordance the post-WMAP $2 \sigma$ region $0.094<\Omega_{\mathrm{dm}} h^{2}<0.129$ [2] where $\Omega_{\mathrm{dm}}$ is the fraction of today's critical density in DM, and $h=0.72 \pm 0.05$ is the Hubble constant in units of $100 \mathrm{~km} /(\mathrm{s} \mathrm{Mpc})$.

As in Split SUSY, the absence of sleptons in our model greatly decreases the number of decay channels available to the LSP [13, 14. Also similar to Split SUSY is the fact that our higgs can be heavier than in the MSSM (in our case the higgs mass is actually a free parameter), hence new decay channels will be available to it, resulting in a large enhancement of its width especially near the $W W$ and $Z Z$ thresholds. This in turn makes accessible neutralino annihilation via a resonant higgs, decreasing the relic density in regions of the parameter space where this channel is accessible. For a very bino-like LSP this is easily the dominant annihilation channel, allowing the bino density to decrease to an acceptable level. We use a modified version of the DarkSUSY [15] code for our relic abundance calculations, explicitly adding the resonant decay of the heavy higgs to $W$ and $Z$ pairs.

As mentioned in the previous section there are also some differences between our model and Split SUSY that are relevant to this discussion: the first is that the Minimal Model contains no wino equivalent (this feature also distinguishes this model from that in [16], which contains a similar dark matter analysis). The second difference concerns the size of the yukawa couplings which govern this mixing, as well as the annihilation cross-section to higgses. Rather than being tied to the gauge couplings at the SUSY breaking scale, these couplings are limited only by the constraint of perturbativity to the cutoff. This means that the yukawas can be much larger in our model, helping a bino-like LSP to both mix more and annihilate more efficiently. These effects are evident in our results and will be discussed in more detail below.

We will restrict our study of DM relic abundance and direct detection in this model to the case with no $\mathrm{CP}$ violating phase $(\theta=0, \pi)$; we briefly comment on the general case in Section 5 . Our results for different values of the yukawa couplings are shown in Figure 1below, in which we highlight the points in the $\mu-M_{1}$ plane that give rise to a relic density within the cosmological bound. The higgs is relatively heavy $\left(M_{\text {higgs }}=160 \mathrm{GeV}\right)$ in this plot in order to access processes with resonant 
annihilation through an s-channel higgs. As we will explain below the only effect this has is to allow a low mass region for a bino-like LSP with $M_{1} \sim M_{\text {higgs }} / 2$.

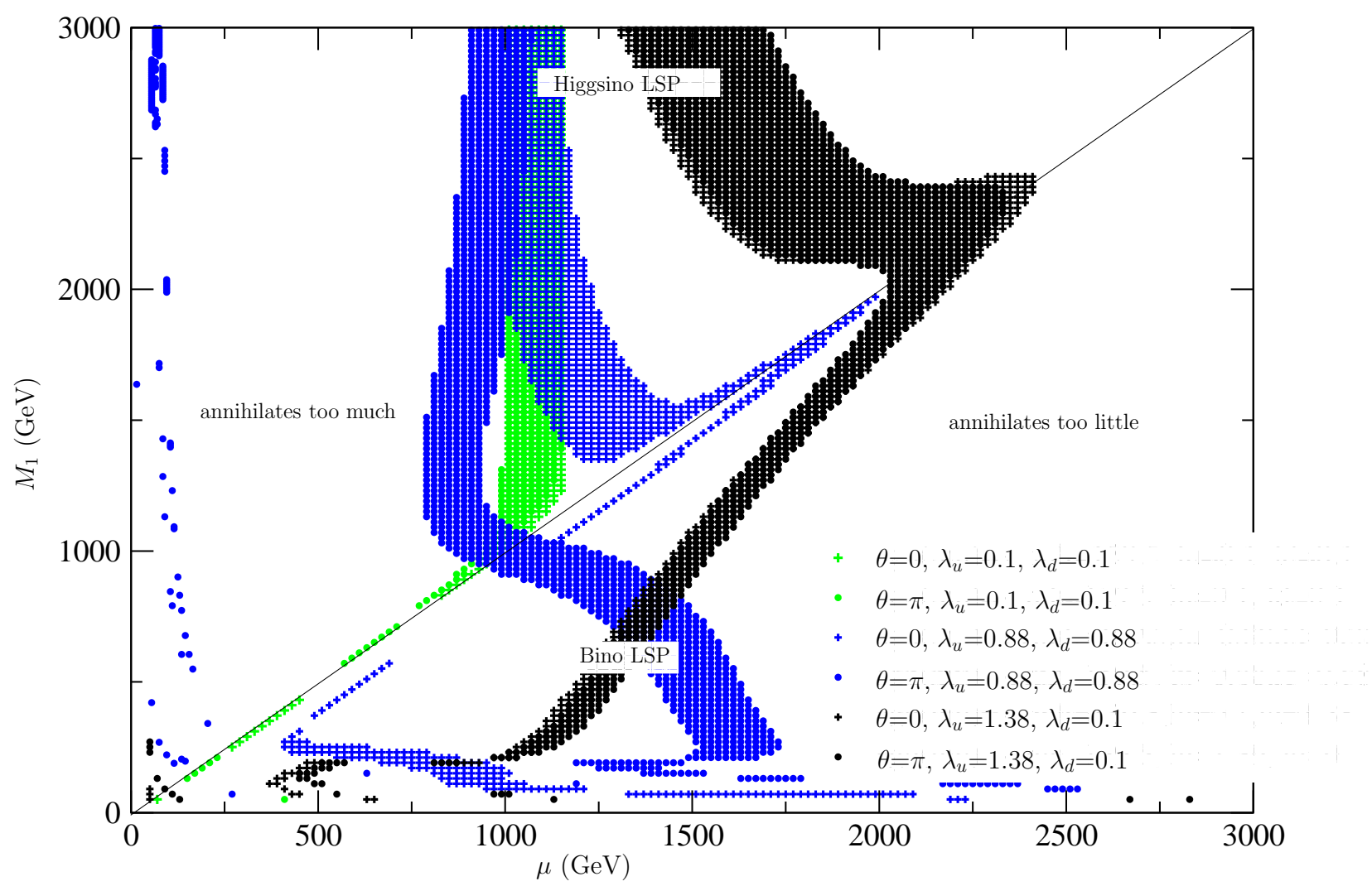

Figure 1: Graph showing regions of parameter space consistent with WMAP.

Notice that the relic abundance seems to be consistent with a dark matter mass as large as 2.2 $\mathrm{TeV}$. Although a detailed analysis of the LHC signature of this model is not within the scope of this paper, it is clear that a large part of this parameter space will be inaccessible at LHC. The pure higgsino region for example, will clearly be hard to explore since the higgsinos are heavy and also very degenerate. There is more hope in the bino LSP region for a light enough spectrum.

While analyzing these results we must keep in mind that $\Omega_{\mathrm{dm}} \sim 10^{-9} \mathrm{GeV}^{-2} /\langle\sigma\rangle_{\text {eff }}$, where $\langle\sigma\rangle_{\text {eff }}$ is an effective annihilation cross section for the LSP at the freeze out temperature, which takes into consideration all coannihilation channels as well as the thermal average [17]. It will be useful to approximate this quantity as the cross-section for the dominant annihilation channel. Although rough, this approximation will help us build some intuition on the behavior of the relic density in different parts of the parameter space. We will not discuss the region close to the origin where the interpretation of the results become more involved due to large mixing and coannihilation.

\subsection{Higgsino Dark Matter}

In order to get a feeling for the structure of Figure 1 it is useful to begin by looking at the regions in which the physics is most simple. This can be achieved by diminishing the the number of annihilation channels that are available to the LSP by taking the limit of small yukawa couplings.

For $\theta=0$, mixing occurs only on the diagonal $M_{1}=\mu$ to a very good approximation (see Appendix $\mathrm{A}$ and Figure 2), hence the region above the diagonal corresponds to a pure higgsino LSP 


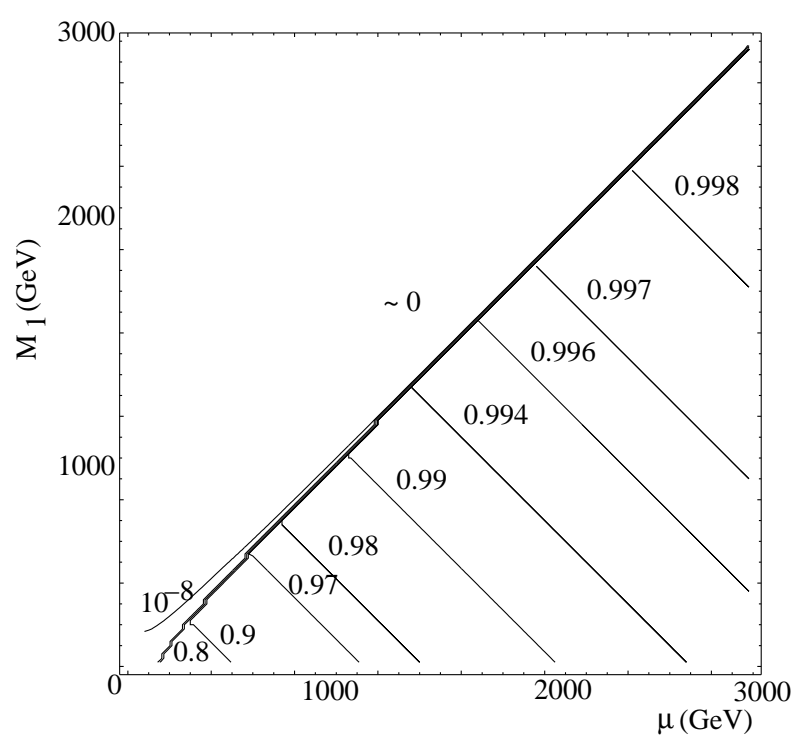

(a) $\theta=0$

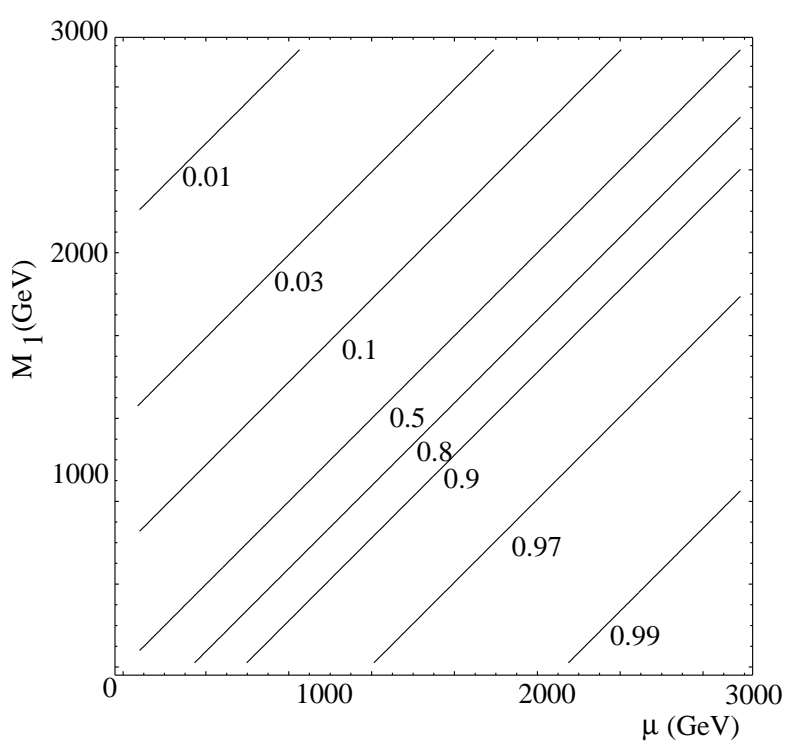

(b) $\theta=\pi$

Figure 2: Gaugino fraction contours for $\lambda_{u}=\lambda_{d}=0.88$ and $\theta=0$ (left), $\pi$ (right).

with mass $\mu$. For $\lambda_{u}=\lambda_{d}=0.1$ the yukawa interactions are irrelevant and the LSP dominantly annihilates by t-channel neutral (charged) higgsino exchange to $Z Z(W W)$ pairs. Charginos, which have a tree-level mass $\mu$ and are almost degenerate with the LSP, coannihilate with it, decreasing the relic density by a factor of 3 . This fixes the LSP mass to be around $\mu=1 \mathrm{TeV}$, giving rise to the wide vertical band that can be seen in the figure; for smaller $\mu$ the LSP over-annihilates, for larger $\mu$ it does not annihilate enough.

Increasing the yukawa couplings increases the importance of t-channel bino exchange to higgs pairs. Notice that taking the limit $M_{1} \gg \mu$ makes this new interaction irrelevant, therefore the allowed region converges to the one in which only gauge interactions are effective. Taking this as our starting point, as we approach the diagonal the mass of the bino decreases, causing the t-channel bino exchange process to become less suppressed and increasing the total annihilation cross-section. This explains the shift to higher masses, which is more pronounced for larger yukawas as expected and peaks along the diagonal where the higgsino and bino are degenerate and the bino mass suppression is minimal. The increased coannihilation between higgsinos and binos close to the diagonal does not play a large part here since both particles have access to a similar t-channel diagram.

Taking $\theta=\pi$ makes little qualitative difference when either of the yukawas is small compared to $M_{1}$ or $\mu$, since in this limit the angle is unphysical and can be rotated away by a redefinition of the higgsino fields. However we can see in Figure 2 that for large yukawas the region above the diagonal $M_{1}=\mu$ changes to a mixed state, rather than being pure higgsino as before. Starting again with the large $M_{1}$ limit and decreasing $M_{1}$ decreases the mass suppression of the t-channel bino exchange diagram like in the $\theta=0$ case, but the LSP also starts to mix more with the bino, an effect that acts in the opposite direction and decreases $\langle\sigma\rangle_{\text {eff }}$. This effect happens to outweigh the former, forcing the LSP to shift to lower masses in order to annihilate enough.

With $\theta=\pi$ and yukawas large enough, there is an additional allowed region for $\mu<M_{W}$. In this region the higgsino LSP is too light to annihilate to on-shell gauge bosons, so the dominant annihilation channels are phase-space suppressed. Furthermore if the splitting between the chargino 
and the LSP is large enough, the effect of coannihilation with the chargino into photon and onshell $W$ is Boltzmann suppressed, substantially decreasing the effective cross-section, and giving the right relic abundance even with such a light higgsino LSP. Although acceptable from a cosmological standpoint, this region is excluded by direct searches since it corresponds to a chargino that is too light.

\subsection{Bino Dark Matter}

The region below the diagonal $M_{1}=\mu$ corresponds to a bino-like LSP. Recall that in the absence of yukawa couplings pure binos in this model do not couple to anything and hence cannot annihilate at all. Turning on the yukawas allows them to mix with higgsinos which have access to gauge annihilation channels. For $\lambda_{u}=\lambda_{d}=0.1$ this effect is only large enough when $M_{1}$ and $\mu$ are comparable (in fact when they are equal, the neutralino states are maximally mixed for arbitrarily small off-diagonal terms), explaining the stripe near the diagonal in Figure 11 Once $\mu$ gets larger than $\sim 1 \mathrm{TeV}$ even pure higgsinos are too heavy to annihilate efficiently; this means that mixing is no longer sufficient to decrease the dark matter relic density to acceptable values and the stripe ends.

Increasing the yukawas beyond a certain value $\left(\lambda_{u}=\lambda_{d}=0.88\right.$, which is slightly larger than their values in Split SUSY, is enough), makes t-channel annihilation to higgses become large enough that a bino LSP does not need to mix at all in order to have the correct annihilation cross-section. This gives rise to an allowed region which is in the shape of a stripe, where for fixed $M_{1}$ the correct annihilation cross-section is achieved only for the small range of $\mu$ that gives the right t-channel suppression. As $M_{1}$ increases the stripe converges towards the diagonal in order to compensate for the increase in LSP mass by increasing the cross-section. Once the diagonal is reached this channel cannot be enhanced any further, and there is no allowed region for heavier LSPs. In addition the cross-section for annihilation through an s-channel resonant higgs, even though CP suppressed (see Section [5 for details), becomes large enough to allow even LSPs that are very pure bino to annihilate in this way. The annihilation rate for this process is not very sensitive to the mixing, explaining the apparent horizontal line at $M_{1}=\frac{1}{2} M_{\text {higgs }} \sim 80 \mathrm{GeV}$. This line ends when $\mu$ grows to the point where the mixing is too small.

As in the higgsino case, taking $\theta=\pi$ changes the shape of the contours of constant gaugino fraction and spreads them out in the plane (see Figure 21), making mixing with higgsinos relevant throughout the region. For small $M_{1}$, the allowed region starts where the mixing term is small enough for the combination of gauge and higgs channels not to cause over-annihilation. Increasing $M_{1}$ again makes the region move towards the diagonal, where the increase in LSP mass is countered by increasing the cross-section for the gauge channel from mixing more.

For either yukawa very large $\left(\lambda_{u}=1.38, \lambda_{d}=0.1\right)$, annihilation to higgses via t-channel higgsinos is so efficient that this process alone is sufficient to give bino-like LSPs the correct abundance. As $M_{1}$ increases the allowed region again moves towards the diagonal in such a way as to keep the effective cross-section constant by decreasing the higgsino mass suppression, thus compensating for the increase in LSP mass. As we remarked earlier since $\lambda_{d}$ is effectively zero in this case, the angle $\theta$ is unphysical and can be rotated away by a redefinition of the higgsino fields.

\section{Direct Detection}

Dark matter is also detectable through elastic scatterings off ordinary matter. The direct detection cross-section for this process can be divided up into a spin-dependent and a spin-independent part; 
we will concentrate on the former since it is usually dominant. As before we restrict to $\theta=0$ and $\pi$, we expect the result not to change significantly for intermediate values.

The spin-independent interaction takes place through higgs exchange, via the yukawa couplings which mix higgsinos and binos. Since the only $\chi_{1}^{0} \chi_{1}^{0} h$ term in our model involves the product of the gaugino and higgsino fractions, the more mixed our dark matter is the more visible it will be to direct detection experiments. This effect can be seen in Fig 3 below.

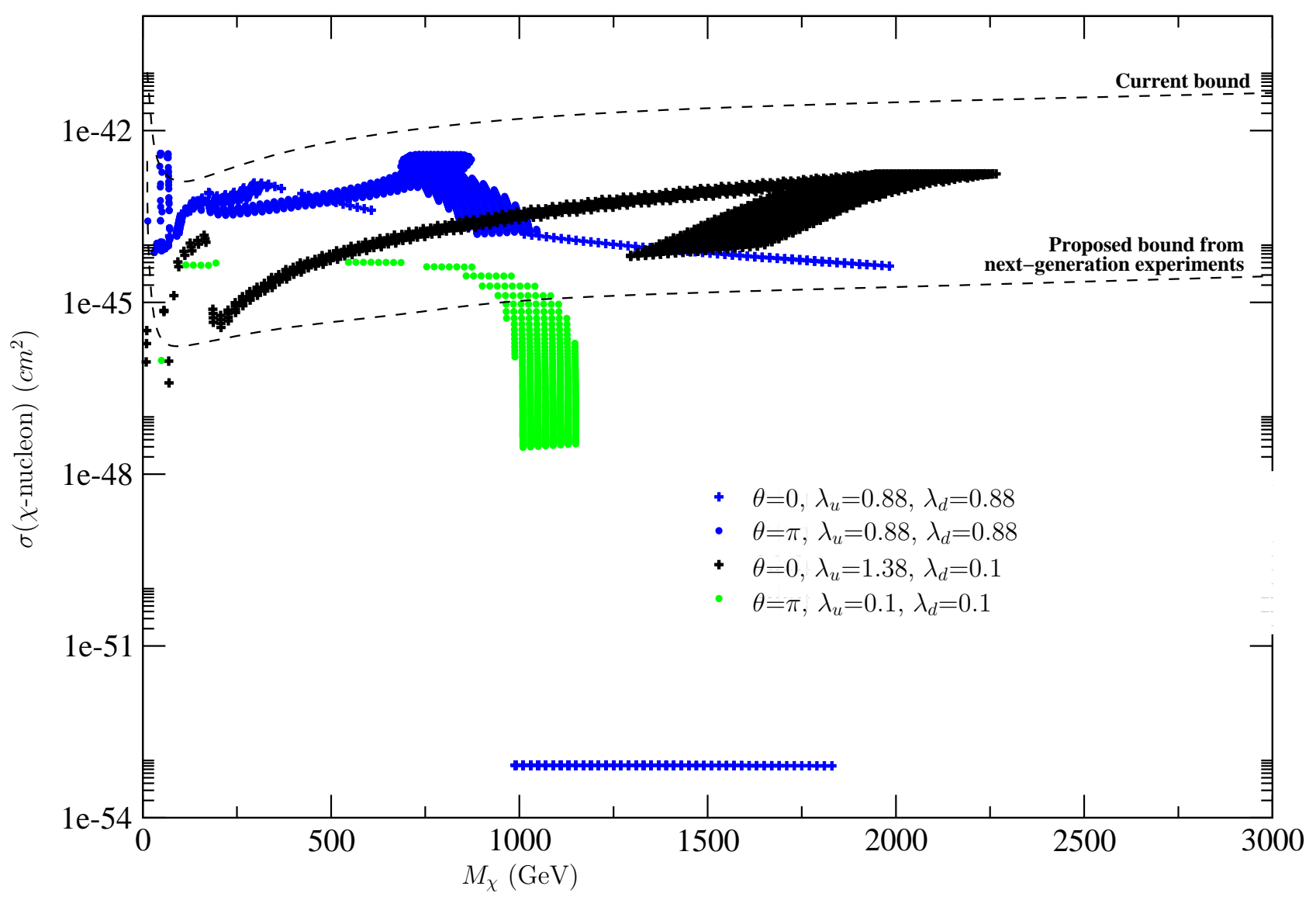

Figure 3: Spin-independent part of dark matter direct detection cross-section. The current bound represents the CDMS limit [1], and, as an indicative value for the proposed bound from next generation experiments, we take the projected sensitivity of SuperCDMS phase B [18].

Although it seems like we cannot currently use this measure as a constraint, the major proportion of our parameter space will be accessible at next-generation experiments. Since higgsino LSPs are generally more pure than bino-type ones, the former will escape detection as long as there is an order $100 \mathrm{keV}$ splitting between its two neutral components. This is is necessary in order to avoid the limit from spin-independent direct detection measurements [12].

Also visible in the graph are the interesting discontinuities mentioned in 13, corresponding to the opening up of new annihilation channels at $M_{L S P}=1 / 2 M_{\text {higgs }}$ through an s-channel higgs. We also notice a similar discontinuity at the top threshold from annihilation to $t \bar{t}$; this effect becomes more pronounced as the new yukawa couplings increase. 


\section{$5 \quad$ Electric Dipole Moment}

Since our model does not contain any sleptons it induces an electron EDM only at two loops, proportional to $\sin (\theta)$ for $\theta$ as defined above. This is a two-loop effect, we therefore expect it to be close to the experimental bound for $\mathcal{O}(1) \theta$. The dominant diagram responsible for the EDM is generated by charginos and neutralinos in a loop and can be seen in Figure 4 below. This diagram is also present in Split SUSY where it gives a comparable contribution to the one with only charginos in the loop [19, 20].

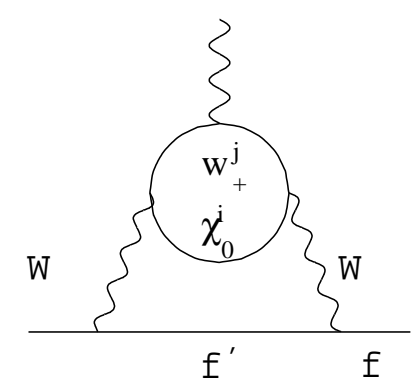

Figure 4: The 2-loop contribution to the EDM of a fermion $\mathrm{f}$.

The induced EDM is (see [19]):

$$
\frac{d_{f}^{W}}{e}= \pm \frac{\alpha^{2} m_{f}}{8 \pi^{2} s_{W}^{4} M_{W}^{2}} \sum_{i=1}^{3} \frac{m_{\chi_{i}} \mu}{M_{W}^{2}} \operatorname{Im}\left(O_{i}^{L} O_{i}^{R *}\right) \mathcal{G}\left(r_{i}^{0}, r^{ \pm}\right)
$$

where

$$
\begin{aligned}
\mathcal{G}\left(r_{i}^{0}, r^{ \pm}\right) & =\int_{0}^{\infty} d z \int_{0}^{1} \frac{d \gamma}{\gamma} \int_{0}^{1} d y \frac{y z(y+z / 2)}{(z+y)^{3}\left(z+K_{i}\right)} \\
& =\int_{0}^{1} \frac{d \gamma}{\gamma} \int_{0}^{1} d y y\left[\frac{\left(y-3 K_{i}\right) y+2\left(K_{i}+y\right) y}{4 y\left(K_{i}-y\right)^{2}}+\frac{K_{i}\left(K_{i}-2 y\right)}{2\left(K_{i}-y\right)^{3}} \ln \frac{K_{i}}{y}\right]
\end{aligned}
$$

and

$$
\begin{aligned}
& K_{i}=\frac{r_{i}^{0}}{1-\gamma}+\frac{r^{ \pm}}{\gamma}, \quad r^{ \pm} \equiv \frac{\mu^{2}}{M_{W}^{2}}, \quad r_{i}^{0} \equiv \frac{m_{\chi_{i}}^{2}}{M_{W}^{2}} \\
& O_{i}^{R}=\sqrt{2} N_{2 i}^{*} \exp ^{-i \theta}, \quad O_{i}^{L}=-N_{3 i}
\end{aligned}
$$

$N^{T} M_{N} N=\operatorname{diag}\left(\mathrm{m}_{\chi_{1}}, \mathrm{~m}_{\chi_{2}}, \mathrm{~m}_{\chi_{3}}\right)$ with real and positive diagonal elements. The sign on the righthand side of equation (2) corresponds to the fermion $f$ with weak isospin $\pm \frac{1}{2}$ and $f^{\prime}$ is its electroweak partner.

In principle it should be possible to cross-correlate the region of our parameter space which is consistent with relic abundance measurements, with that consistent with electron EDM measurements in order to further constrain our parameters. However since the current release of DarkSUSY does not support $\mathrm{CP}$ violating phases and a version including $\mathrm{CP}$ violations seems almost ready for public release ${ }^{4}$ we leave an accurate study of the consequences of non-zero CP phase in relic abundance and direct detection calculations for a future work. We can still draw some interesting

\footnotetext{
${ }^{4}$ Private communication with one of the authors of DarkSUSY.
} 
conclusions by estimating the effect of non-zero CP phase. Because there is no reason for these new contributions to be suppressed with respect to the CP-conserving ones (for $\theta$ of $\mathcal{O}(1)$ ), we might naively expect their inclusion to enhance the annihilation cross-section by around a factor of 2 , increasing the acceptable LSP masses by $\sim \sqrt{2}$ for constant relic abundance. This is discussed in greater detail in [21] (and 22] for direct detection) in which we see that this observation holds for most of the parameter space. We must note, however, that in particular small regions of the space the enhancement to the annihilation cross-section and the suppression to the elastic cross section can be much larger, justifying further investigation of this point in future work. With this assumption in mind we see in Figure [5 that although the majority of our allowed region is below the current experimental limit of $d_{e}<1.7 \times 10^{-27} e \mathrm{~cm}$ at $95 \%$ C.L. [23, most of it will be accessible to next generation EDM experiments. These propose to improve the precision of the electron EDM measurement by 4 orders of magnitude in the next 5 years, and maybe even up to 8 orders of magnitude, funding permitting [24, 25, 26]. We also see in this figure that $\mathrm{CP}$ violation is enhanced on the diagonal where the mixing is largest. This is as expected since the yukawas that govern the mixing are necessary for there to be any $\mathrm{CP}$ violating phase at all. For the same reason, decoupling either particle sends the EDM to zero.

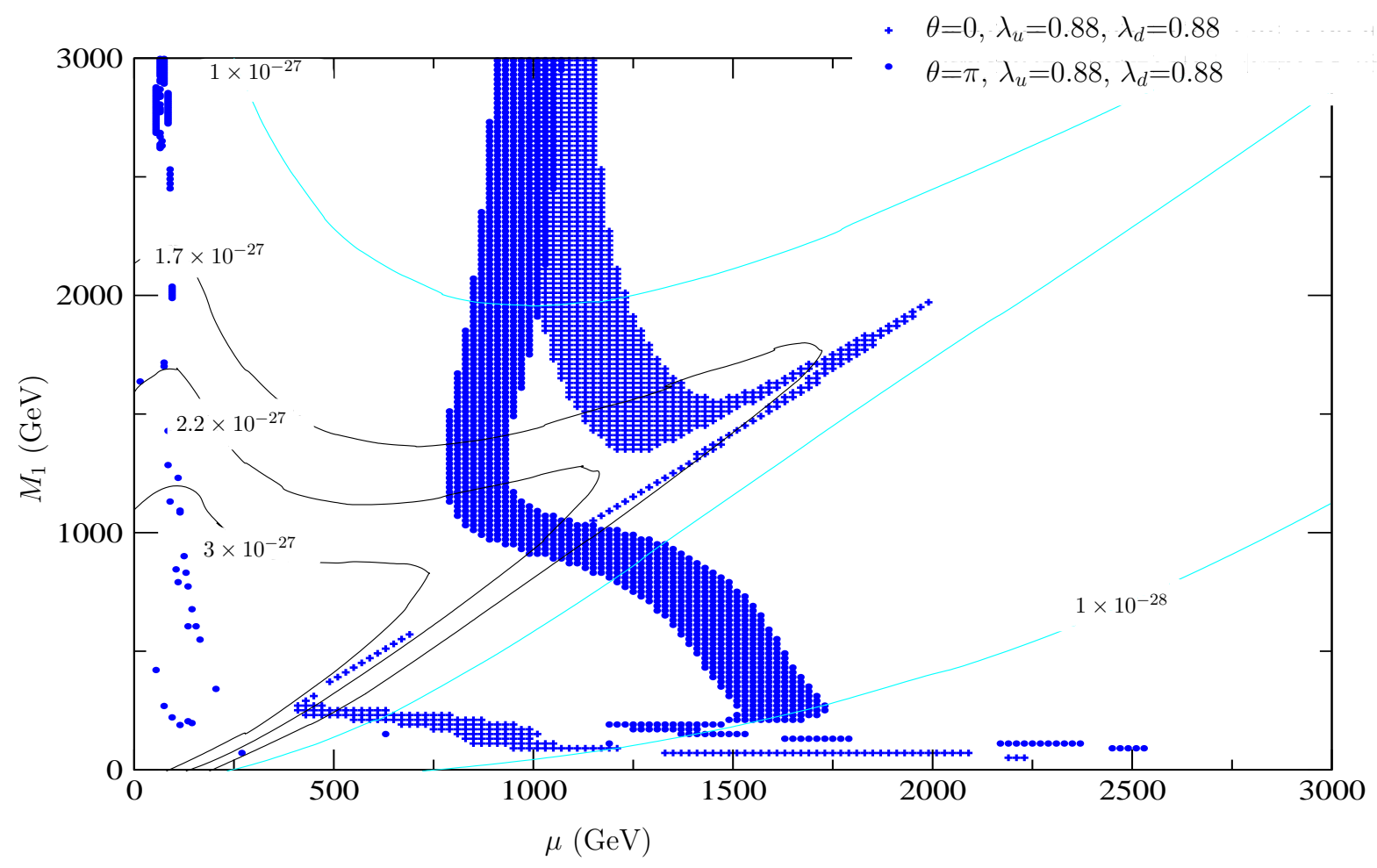

Figure 5: Electron edm contours for $\theta=\pi / 2$. The excluded region is bounded by the black contours. Note that $\mathrm{CP}$ violation was not included in the relic density calculation, and the dark matter plot is simply intended to indicate the approximate region of interest for dark matter.

\section{Gauge Coupling Unification}

In this section we study the running of gauge couplings in our model at two loops. The addition of higgsinos largely improves unification as compared to the SM case, but their effect is still not large 
enough and the model predicts a value for $\alpha_{s}\left(M_{z}\right)$ around $9 \sigma$ lower than the experimental value of $\alpha_{s}\left(M_{z}\right)=0.119 \pm 0.002$ [27]. Moreover the scale at which the couplings unify is very low, around $10^{14} \mathrm{GeV}$, making proton decay occur much too quickly to embed in a simple GUT theory ${ }^{5}$. These problems can be avoided by adding the Split SUSY particle content at higher energies, as in [29], at the cost of losing minimality; instead we choose to solve this problem by embedding our minimal model in an extra dimensional theory. This decision is well motivated: even though normal 4D GUTs have had some successes, explaining the quark-lepton mass relations for example, and charge quantization in the SM, there are many reasons why these simple theories are not ideal. In spite of the fact that the matter content of the SM falls naturally into representations of $S U(5)$, there are some components that seem particularly resistant to this. This is especially true of the higgs sector, the unification of which gives rise to the doublet-triplet splitting problem. Even in the matter sector, although $b-\tau$ unification works reasonably well the same cannot be said for unification of the first two generations. In other words, it seems like gauge couplings want to unify while the matter content of the SM does not, at least not to the same extent. This dilemma is easily addressed in an extra dimensional model with a GUT symmetry in the bulk, broken down to the SM gauge group on a brane by boundary conditions [30] since we can now choose where we put fields based on whether they unify consistently or not. Unified matter can be placed in the bulk whereas non-unified matter can be placed on the GUT-breaking brane. The low energy theory will then contain the zero modes of the 5D bulk fields as well as the brane matter. While solving many of the problems of standard 4D GUTs these extra dimensional theories have the drawback of having a large number of discrete choices for the location of the matter fields, as we shall see later.

We will consider a model with one flat extra dimension compactified on a circle of radius $R$, with orbifolds $S^{1} /\left(Z_{2} \times Z_{2}^{\prime}\right)$, whose action is obtained from the identifications

$$
Z_{2}: y \sim 2 \pi R-y, \quad Z_{2}^{\prime}: y \sim \pi R-y, \quad y \in[0,2 \pi]
$$

where $y$ is the coordinate of the fifth dimension. There are two fixed points under this action, at $(0, \pi R)$ and $(\pi R / 2,3 \pi R / 2)$, at which are located two branes. We impose an $S U(5)$ symmetry in the bulk and on the $y=0$ brane; this symmetry is broken down to the SM $S U(3) \times S U(2) \times U(1)$ on the other brane by a suitable choice of boundary conditions. All fields need to have definite transformation properties under the orbifold action - we choose the action on the fundamental to be $\phi \rightarrow \pm P \phi$ and on the adjoint, $\pm[P, A]$, for projection operators $P_{Z}=(+,+,+,+,+)$ and $P_{Z^{\prime}}=(+,+,+,-,-)$. This gives SM gauge fields and their corresponding KK towers $A_{\mu}^{a}$ for $a=\{1, \ldots, 12\}(+,+)$ parity; and the towers $A_{\mu}^{\hat{a}}$ for $\hat{a}=\{13, \ldots, 24\}(+,-)$ parity, achieving the required symmetry-breaking pattern. By gauge invariance the unphysical fifth component of the gauge field, which is eaten in unitary gauge, gets opposite boundary conditions. ${ }^{6}$ We still have the freedom to choose the location of the matter fields. In this model $S U(5)$-symmetric matter fields in the bulk will get split by the action of the $Z^{\prime}$ orbifold: the SM $\overline{\mathbf{5}}$ for instance will either contain a massless $d^{c}$ or a massless $l$, with the other component only having massive modes. Matter fields in the bulk must therefore come in pairs with opposite eigenvalues under the orbifold projections,

\footnotetext{
${ }^{5}$ It is possible to evade the constraint from proton decay by setting some of the relevant mixing parameters to zero [28. However we are not aware of any GUT model in which such an assertion is justified by symmetry arguments.

${ }^{6}$ From an effective field theory point of view an orbifold is not absolutely necessary, our theory can simply be thought of as a theory with a compact extra dimension on an interval, with two branes on the boundaries. Because of the presence of the boundaries we are free to impose either Dirichlet or Neumann boundary conditions for the bulk field on each of the branes breaking the $S U(5)$ to the SM gauge group purely by choice of boundary conditions and similarly splitting the multiplets accordingly. Our orbifold projection is therefore nothing more than a further restriction to the set of all possible choices we can make.
} 
so for each SM generation in the bulk we will need two copies of $\mathbf{1 0}+\overline{\mathbf{5}}$. This provides us with a simple mechanism to forbid proton decay from $X$ - and $Y$-exchange and also to split the color triplet higgs field from the doublet. To summarize, unification of SM matter fields in complete multiplets of $S U(5)$ cannot be achieved in the bulk but on the $S U(5)$ brane, while matter on the SM brane is not unified into complete GUT representations.

\subsection{Running and matching}

We run the gauge couplings from the weak scale to the cutoff $\Lambda$ by treating our model as a succession of effective field theories (EFTs) characterized by the differing particle content at different energies. The influence of the yukawa couplings between the higgsinos and the singlet on the two-loop running is negligible, hence it is fine to assume that the singlet is degenerate with the higgsinos so there is only one threshold from $M_{\text {top }}$ to the compactification scale $1 / R$, at which we will need to match with the full $5 \mathrm{D}$ theory.

The $S U(5)$-symmetric bulk gauge coupling $g_{5}$ can be matched on to the low energy couplings at the renormalization scale $M$ via the equation

$$
\frac{1}{g_{i}^{2}(M)}=\frac{2 \pi R}{g_{5}^{2}}+\Delta_{i}(M)+\lambda_{i}(M R)
$$

The first term on the right represents a tree level contribution from the $5 \mathrm{~d}$ kinetic term, $\Delta_{i}$ are similar contributions from brane-localized kinetic terms and $\lambda_{i}$ encode radiative contributions from KK modes. The latter come from renormalization of the $4 \mathrm{D}$ brane kinetic terms which run logarithmically as usual.

To understand this in more detail let us consider radiative corrections to a $U(1)$ gauge coupling in an extra dimension compactified on a circle with no orbifolds, due to a 5D massless scalar field 32. Since $1 / g_{5}^{2}$ has mass dimension 1 , by dimensional analysis we might expect corrections to it to go like $\Lambda+m \log \Lambda$ where $m$ is some mass parameter in the theory. The linearly divergent term is UV sensitive and can be reabsorbed into the definition of $g_{5}$, whereas the log term cannot exist since there is no mass parameter in the theory. Hence the 5D gauge coupling does not run, and neither does the $4 \mathrm{D}$ gauge coupling. This can also be interpreted from a $4 \mathrm{D}$ point of view, where the KK partners of the scalar cut off the divergences of the zero mode. Since there is no distinction between the wavefunctions for even (cosine) and odd (sine) KK modes in the absence of an orbifold, and we know that the sum of their contributions must cancel the log divergence of the 4D massless scalar, each of these must give a contribution equal to $-1 / 2$ times that of the $4 \mathrm{D}$ massless scalar.

When we impose a $Z_{2}$ orbifold projection and add two 3-branes at the orbifold fixed points, the scalar field must now transform as an eigenstate of this orbifold action and can either be even $((+,+)$, with Neuman boudary conditions on the branes), or odd $((-,-)$, with Dirichlet boundary conditions on the branes). This restricts us to a subset of the original modes and the cancellation of the log divergence no longer works. Since this running can only be due to $4 \mathrm{D}$ gauge kinetic terms localized on the branes, where the gauge coupling is dimensionless and can therefore receive logarithmic corrections, locality implies that the contribution from a tower of states on a particular brane can only be due to its boundary condition on that brane, with the total running equal to the sum of the contributions on each brane. In fact, it is only in the vicinity of the brane that imposing a particular boundary condition has any effect. As argued above, a $(+,+)$ tower (excluding the zero mode) and a $(-,-)$ tower must each give a total contribution equal to $-1 / 2$ times that of the zero mode, which corresponds to a coefficient of $-1 / 4$ to the running of each brane-localized kinetic term. Taking into account the contribution of the zero mode we can say that a tower of modes with

+ boundary conditions on a brane contributes $+1 / 4$ times the corresponding $4 \mathrm{D}$ coefficient, while a 
- boundary condition contributes $-1 / 4$ times the same quantity. This argument makes it explicit that the orbifold projection can be seen as a prescription on the boundary conditions of the fields in the extra dimension, which only affect the physics near each brane.

Adding another orbifold projection as we are doing in this case also allows for towers with $(+,-)$ and $(-,+)$ boundary conditions which, from the above argument, both give a contribution of $\pm 1 / 4 \mp 1 / 4=0$. The contribution of the $(+,+)$ and $(-,-)$ towers clearly remains unchanged.

Explicitly integrating out the KK modes at one loop at the compactification scale allows us to verify this fact, and also compute the constant parts of the threshold corrections, which are scheme-dependent. In $\overline{\mathrm{DR}} 7$ we obtain [32]:

$$
\lambda_{i}(M R)=\frac{1}{96 \pi^{2}}\left(\left(b_{i}^{S}-21 b_{i}^{G}+8 b_{i}^{F}\right) F_{e}(M R)+\left(\tilde{b}_{i}^{S}-21 \tilde{b}_{i}^{G}+8 \tilde{b}_{i}^{F}\right) F_{0}\right)
$$

with

$$
\begin{aligned}
& F_{e}(\mu R)=\mathcal{I}-1-\log (\pi)-\log (M R), \quad F_{0}=-\log (2) \\
& \mathcal{I}=\frac{1}{2} \int_{1}^{+\infty} d t\left(t^{-1}+t^{-1 / 2}\right)\left(\theta_{3}(i t)-1\right) \simeq 0.02, \quad \theta_{3}(i t)=\sum_{n=-\infty}^{+\infty} e^{-\pi t n^{2}}
\end{aligned}
$$

where $b_{i}^{S, G, F}\left(\tilde{b}_{i}^{S, G, F}\right)$ are the Casimirs of the KK modes of real scalars (not including goldstone bosons), massive vector bosons and Dirac fermions respectively with even (odd) masses $2 n / R((2 n+$ $1) / R)$. As explained above, the logarithmic part of the above expression is equal to exactly $-1 / 2$ times the contribution of the same fields in $4 \mathrm{D}$ [33, 34]. Since the compactification scale $1 / R$ will always be relatively close to the unification scale $\Lambda$ (so our $5 \mathrm{D}$ theory remains perturbative), it will be sufficient for us to use one loop matching in our two loop analysis as long as the matching is done at a scale $M$ close to the compactification scale.

As an aside, from equation (4) we can get:

$$
\frac{d}{d t} \Delta_{i}=\frac{b_{i}-b_{i}^{M M}}{8 \pi^{2}}
$$

where $b_{i}$ is shorthand for the combination $\left(b_{i}^{S}-21 b_{i}^{G}+8 b_{i}^{F}\right) / 12$ and $b_{i}^{M M}$ are the coefficients of the renormalization group equations below the compactification scale (see Appendix B for details). It is clear from this equation that it is unnatural to require $\Delta_{i}(1 / R) \ll 1 /\left(8 \pi^{2}\right)$. The most natural assumption $\Delta_{i}(\Lambda) \sim 1 /\left(8 \pi^{2}\right)$ gives a one-loop contribution comparable to the tree level term, implying the presence of some strong dynamics in the brane gauge sector at the scale $\Lambda$. We know that the $5 \mathrm{D}$ gauge theory becomes strong at the scale $24 \pi^{3} / g_{5}^{2}$, so from naive dimensional analysis (NDA) (see for example [35]) we find that it is quite natural for $\Lambda$ to coincide with the strong coupling scale for the bulk gauge group.

Running equation (7) to the compactification scale we obtain

$$
\Delta_{i}(1 / R)=\Delta_{i}(\Lambda)+\frac{b_{i}-b_{i}^{M M}}{8 \pi^{2}} \log (\Lambda R)
$$

For $\Lambda R \gg 1$ the unknown bare parameter is negligible compared to the log-enhanced part, and can be ignored, leaving us with a calculable correction. Using $g_{G U T}=2 \pi R / g_{5}^{2}$ we expect $\Lambda R \sim$ $8 \pi^{2} / g_{\text {GUT }}^{2} \sim 100$. Keeping this in mind, we shall check whether unification is possible in our model

\footnotetext{
${ }^{7}$ We use this renormalization scheme even though our theory is non-supersymmetric since $4 \mathrm{D}$ threshold corrections in this scheme contain no constant part [31.
} 
with $\Lambda R$ in the regime where the bare brane gauge coupling is negligible. To this purpose we will impose the matching equation (44) at the scale $\Lambda$ assuming $\Delta_{i}(\Lambda)=0$; we will then check whether the value of $\Lambda R$ found justifies this approximation.

In order to develop some intuition for the direction that these thresholds go in, we can analyze the one-loop expression (with one-loop thresholds) for the gauge couplings at $M_{z}$ :

$$
\frac{1}{\alpha_{i}\left(M_{z}\right)}=\frac{4 \pi}{g_{\mathrm{GUT}}^{2}}+4 \pi \lambda_{i}(\Lambda R)+\lambda_{i}^{\mathrm{conv}}(\Lambda R)+\frac{b_{i}^{\mathrm{MM}}}{2 \pi} \log \left(\frac{\Lambda}{M_{z}}\right)+\frac{\left(b_{i}^{\mathrm{SM}}-b_{i}^{\mathrm{MM}}\right)}{2 \pi} \log \left(\frac{\mu}{M_{z}}\right)
$$

$b_{i}^{\mathrm{SM}}$ are the SM beta function coefficients (see Appendix $(\mathrm{B}), \mu$ is the scale of the higgsinos and singlet, $\lambda^{\text {conv }}=\left(-\frac{3}{12 \pi},-\frac{2}{12 \pi}, 0\right)$ are conversion factors from $\overline{\mathrm{MS}}$, in which the low-energy experimental values for the gauge couplings are defined, to $\overline{\mathrm{DR}}\left[36\right.$. Taking the linear combination $(9 / 14) \alpha_{1}^{-1}-$ $(23 / 14) \alpha_{2}^{-1}+\alpha_{3}^{-1}$ allows us to eliminate the $\Lambda$ dependence as well as all $S U(5)$-symmetric terms, leaving

$$
\frac{1}{\alpha_{3}\left(M_{z}\right)}=-\frac{9 / 14}{\alpha_{1}\left(M_{z}\right)}+\frac{23 / 14}{\alpha_{2}\left(M_{z}\right)}+4 \pi \lambda(\Lambda R)+\lambda^{\mathrm{conv}}+\frac{\left(b^{\mathrm{SM}}-b^{\mathrm{MM}}\right)}{2 \pi} \log \left(\frac{\mu}{M_{z}}\right)
$$

where $X=(9 / 14) X_{1}-(23 / 14) X_{2}+X_{3}$ for any quantity $X$. Recall that the leading threshold correction from the $5 \mathrm{D}$ GUT is proportional to $\log (\Lambda R)$. The low-energy value of $\alpha_{3}$ is therefore changed by

$$
\delta \alpha_{3}\left(M_{z}\right)=\alpha_{3}\left(M_{z}\right)^{2} \frac{b}{2 \pi} \log (\Lambda R)
$$

We still have the freedom to choose the positions of the various matter fields. In order to determine the best setup for gauge coupling unification we need to keep in mind two facts: the first is that adding $S U(5)$ multiplets in the bulk does not have any effect on $\alpha_{3}\left(M_{Z}\right)$; and the second is that $b$ contains only contributions from $(+,+)$ modes (in unitary gauge none of our bulk modes have $(-,-)$ boundary conditions; our $S U(5)$ bulk multiplets are split into $(+,+)$ and $(+,-)$ modes).

As stated at the beginning of this section our $4 \mathrm{D}$ prediction for $\alpha_{3}\left(M_{z}\right)$ is too low. Since fermions have a larger effect on running than scalars, this problem is most efficiently tackled by splitting up the fermion content of the SM into non-SU(5) symmetric parts in order to make $b$ as positive as possible. Examining the particular linear combination that eliminated the dependence on $\Lambda$ at one loop we find that one or more $S U(5)$-incomplete colored multiplets are needed in the bulk, or equivalently the weakly-interacting part of the same multiplet has to be on one of the branes. Since matter in the bulk is naturally split by the orbifold projections, this just involves separating the pair of multiplets whose zero modes make up one SM family.

With this in mind we find that for fixed $\Lambda R$ and $\mu$, since separating different numbers of SM generations allows us to vary the low energy value of $\alpha_{3}$ anywhere from its experimental value to several $\sigma$ s off, gauge coupling unification really does work in this model for some fraction of all available configurations. Although this may seem a little unsatisfactory from the point of view of predictivity, the situation can be somewhat ameliorated by further refining our requirements. For example, we can go some way towards explaining the hierarchy between the SM fermion masses by placing the first generation in the bulk, the second generation split between the bulk and a brane and the third generation entirely on a brane. This way, in addition to breaking the approximate flavor symmetry in the fermion sector we also obtain helpful factors of order $1 / \sqrt{\Lambda R}$ between the masses of the different generations. The location of the higgs does not have a very large effect on unification, the simplest choice would be to put it, as well as the higgsinos and singlet, on the 
$S U(5)$-breaking brane, where there is no need to introduce corresponding color triplet fields. This also helps to explain the hierarchy. In this model, which can be seen on the left-hand side of Figure

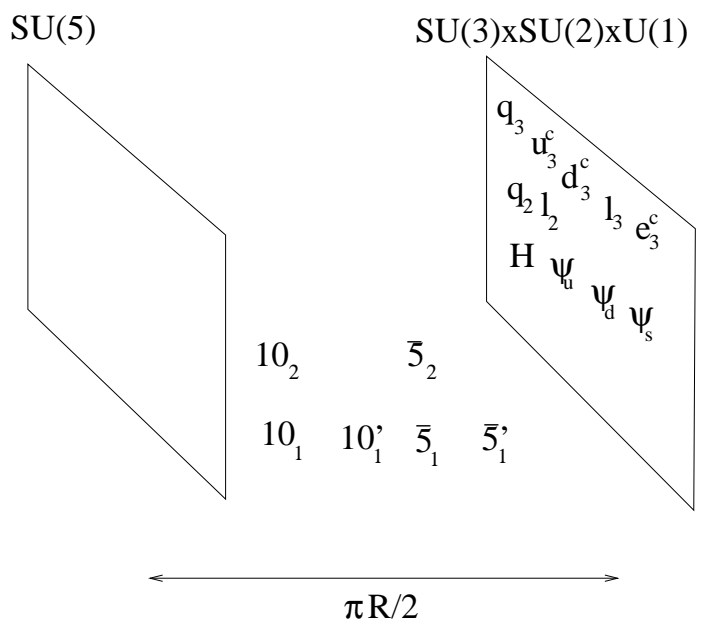

(a) Higgs on the broken brane

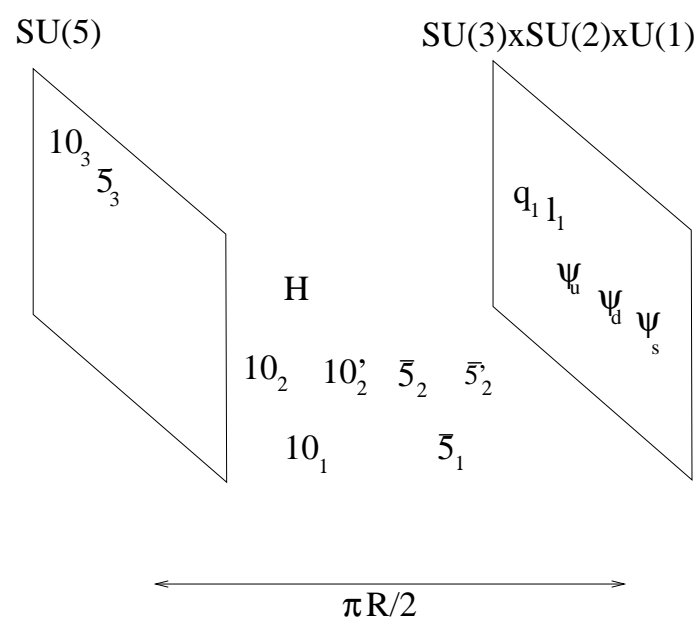

(b) Higgs in the bulk and third generation on the $S U(5)$ brane

Figure 6: Matter content of the two orbifold GUT models we propose.

6. we also need to put our third generation and split second generation on the same brane in order for them to interact with the higgs.

On the right is another model which capitalizes on every shred of evidence we have about GUT physics: we put the higgs in the bulk in this case (recall that the orbifold naturally gives rise to doublet-triplet splitting) so that we can switch the third generation to the $S U(5)$-preserving brane and obtain $b-\tau$ unification (see Figure 17) without having analogous relationships for the other two generations $^{8}$. We also need to flip the positions of the first and second generations if we want to keep the suppression of the mass of the first generation with respect to the second.

The low-energy values for $\alpha_{3}$ as a function of $\mu$ in these two models can be seen in Figure 8 for different $\Lambda R$. Note that unification can be acheived in the regime where $\Lambda R \gg 1$, justifying our initial assumption that the brane kinetic terms could be neglected. We see that although the dependence on $\mu$ is very slight, small $\mu$ seems to be preferred. However we cannot use this observation to put a firm upper limit on $\mu$ because of the uncertainties associated with ignoring the bare kinetic terms on the branes.

The second configuration, Figure 6(b) also gives proton decay through the mixing of the third generation with the first two. From our knowledge of the CKM matrix we infer that all mixing matrices will be close to the unit matrix, proton decay will therefore be suppressed by off-diagonal elements. To minimize this suppression it is best to have an anti-neutrino and a strange quark in the final state. The proton decay rate for this process was computed in 38, and is proportional to $\left(\frac{g_{4}^{2}}{(1 / R)^{2}}\right)^{2}\left(1-\frac{m_{\text {kaon }}^{2}}{m_{\text {proton }}^{2}}\right)\left|\left(R_{d}^{\dagger}\right)_{23}\left(R_{u}^{\dagger}\right)_{13}\left(L_{d}\right)_{31}\right|^{2}$ where $R_{d, u}$ and $L_{d}$ are the rotation matrices of

\footnotetext{
${ }^{8}$ As explained in [37] the two yukawa couplings $\lambda_{b}$ and $\lambda_{\tau}$ run differently only below the compactification
} scale. Because of locality, the fields living on the $S U(5)$ brane do not feel the $S U(5)$ breaking until energies below the compactification scale; hence if they are unified at some high energy they keep being unified until this scale. 


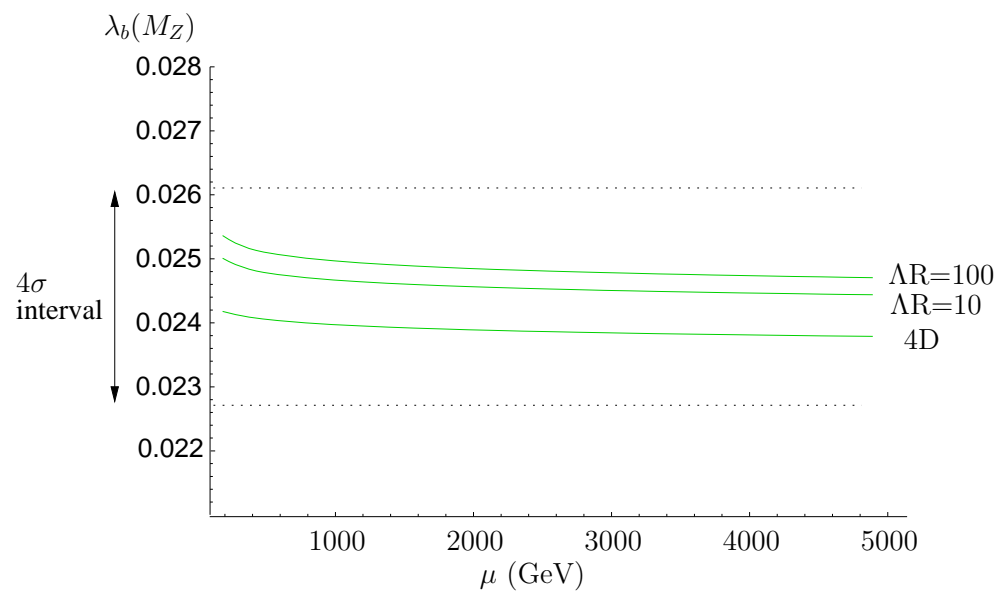

Figure 7: Low energy prediction for $\lambda_{b}\left(M_{Z}\right)$, as a function of the higgsino mass $\mu$, for the model with the higgs in the bulk, for $\Lambda R=10,100$ and for the $4 \mathrm{D}$ model. $4 \sigma$ interval taken from [27].

the right-handed down-type and up-type quarks, and the left-handed down-type quarks, which are unknown. We assume that the 2-3 and the 1-3 mixing elements are 0.05 and 0.01 respectively, similar to the corresponding CKM matrix elements, giving a proton lifetime of

$$
\tau_{p}\left(p \rightarrow K^{+} \bar{\nu}_{\tau}\right) \simeq 6.6 \times 10^{38} \text { years } \times\left(\frac{1 / R}{10^{14} \mathrm{GeV}}\right)^{4} \simeq 4 \times 10^{35} \text { years }
$$

for $1 / R=1.6 \times 10^{13} \mathrm{GeV}$. This is above the current limit from Super-Kamiokande of $1.9 \times 10^{33}$ years at $90 \%$ C.L. 39, 40, although there are multi-megaton experiments in the planning stages that are expected to reach a sensitivity of up to $6 \times 10^{34}$ years [40]. Given our lack of information about the mixing matrices involved ${ }^{9}$, we see that there might be some possibility that proton decay in this model will be seen in the not-too-distant future.

\section{Conclusion}

The identification of a $\mathrm{TeV}$-scale weakly-interacting particle as a good dark matter candidate, and the unification of the gauge couplings are usually taken as indications of the presence of low-energy SUSY. However this might not necessarily be the case.

If we assume that the tuning of the higgs mass can be explained in some other unnatural way, through environmental reasoning for instance, then new possibilities open up for physics beyond the SM. In this paper we studied the minimal model consistent with current experimental limits, that has both a good thermal dark matter candidate and gauge coupling unification. To this end we added to the SM two higgsino-like particles and a singlet, with a singlet majorana mass of $\lesssim 100 \mathrm{TeV}$ in order to split the two neutralinos and so avoid direct detection constraints. Making the singlet light allowed for a new region of dark matter with mixed states as heavy as $\sim 2.2 \mathrm{TeV}$, well beyond the reach of the LHC and the generic expectation for a weakly interacting particle. Nevertheless we do have some handles on this model: firstly via the 2-loop induced electron EDM contribution which is just beyond present limits for $\mathrm{CP}$ angle of order 1 , and secondly by the spin-independent direct detection cross section, both of which should be accessible at next-generation experiments.

\footnotetext{
${ }^{9}$ Experiments have only constrained the particular combination that appears in the SM as the CKM matrix.
} 


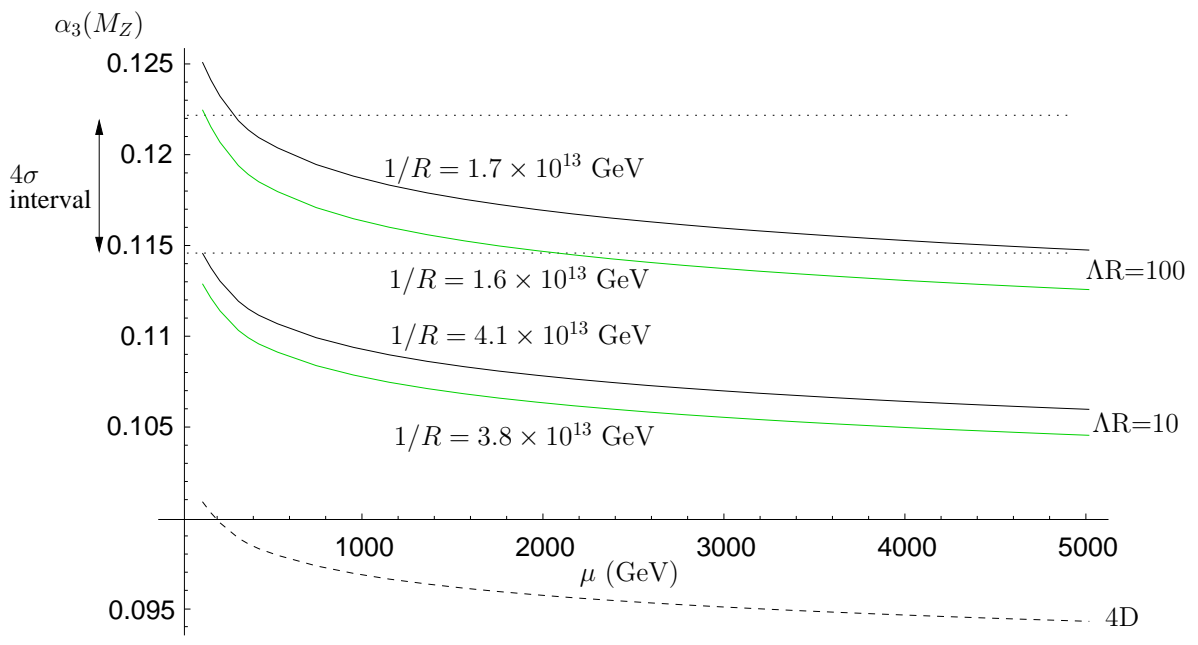

Figure 8: Low energy prediction for $\alpha_{3}\left(M_{z}\right)$, as a function of the higgsino mass $\mu$, for the model with the higgs on the brane (black line), and for the model with the higgs in the bulk (green line), for $\Lambda R=10,100$, and for the $4 \mathrm{D}$ case (dashed line). Some typical values of $1 / R$ are shown.

Turning to gauge coupling unification we saw that this was much improved at two loops by the presence of the higgsinos. A full 4D GUT model is nevertheless excluded by the smallness of the GUT scale $\sim 10^{14} \mathrm{GeV}$, which induces too fast proton decay. We embedded the model in a $5 \mathrm{D}$ orbifold GUT in which the threshold corrections were calculable and pushed $\alpha_{3}$ in the right direction for unification (for a suitable matter configuration). It is very gratifying that such a model can help explain the pattern in the fermion mass hierarchy, give $b-\tau$ unification, and predict a rate for proton decay that can be tested in the future.

\section{Acknowledgments}

First and foremost we would like to thank Nima Arkani-Hamed for his constant guidance and insight. Thanks also to Aaron Pierce for various helpful suggestions; and Paolo Creminelli, Alberto Nicolis, Philip Schuster, Jesse Thaler, and Natalia Toro for useful conversations.

\section{Appendix}

\section{A The neutralino mass matrix}

We have a $3 \times 3$ neutralino mass matrix in which the mixing terms (see equation (2)) are unrelated to gauge couplings and are limited only by the requirement of perturbativity to the cutoff. It is possible to get a feel for the behavior of this matrix by finding the approximate eigenvalues and eigenvectors in the limit of equal and small off-diagonal terms $\left(\lambda v \ll M_{1} \pm \mu\right)$. The approximate eigenvalues and eigenvectors are shown in the table below: 


\begin{tabular}{|c|c|}
\hline$M^{2}$ & Gaugino fraction \\
\hline \hline$M_{1}^{2}+4 \lambda^{2} v^{2} \frac{M_{1}}{M_{1} \pm \mu}$ & $1-2 \frac{\lambda^{2} v^{2}}{\left(M_{1} \pm \mu\right)^{2}}$ \\
\hline$\mu^{2}$ & 0 \\
\hline$\mu^{2} \pm 4 \lambda^{2} v^{2} \frac{\mu}{M_{1} \pm \mu}$ & $2 \frac{\lambda^{2} v^{2}}{\left(M_{1} \pm \mu\right)^{2}}$ \\
\hline
\end{tabular}

for $\cos \theta= \pm 1$.

If $M_{1}<<\mu$ the first eigenstate will be the LSP. In the opposite limit the composition of the LSP is dependent upon the sign of $\cos (\theta)$. For $\cos (\theta)$ positive the second eigenstate, which is pure higgsino, is the LSP, while for $\cos (\theta)$ negative, the mixed third eigenstate becomes the LSP.

We also see from the table that in the pure higgsino case, a splitting of order $100 \mathrm{keV}$ (sufficient to evade the direct detection constraint) can be achieved with a singlet mass lighter than $10^{9} \mathrm{GeV}$, where the upper limit corresponds to $\mathrm{O}(1)$ yukawa couplings.

\section{B Two-Loop Beta Functions for Gauge Couplings}

The two-loop RGE for the gauge couplings in our minimal model is

$$
(-2 \pi) \frac{d}{d t} \alpha_{i}^{-1}=b_{i}^{M M}+\frac{1}{(4 \pi)^{2}}\left[\sum_{j}^{3} 4 \pi B_{i j}^{M M} \alpha_{j}-d_{i} \lambda_{t}^{2}-d_{i}^{\prime}\left(\lambda_{u}^{2}+\lambda_{d}^{2}\right)\right]
$$

with $\beta$-function coefficients

$$
b^{M M}=\left(\frac{9}{2},-\frac{15}{6},-7\right) \quad B^{M M}=\left(\begin{array}{ccc}
\frac{104}{25} & \frac{18}{5} & \frac{44}{5} \\
\frac{6}{5} & 14 & 12 \\
\frac{11}{10} & \frac{9}{2} & -26
\end{array}\right) \quad d=\left(\frac{17}{10}, \frac{3}{2}, 2\right) \quad d^{\prime}=\left(\frac{3}{20}, \frac{1}{4}, 0\right)
$$

The running of the yukawa couplings is the same as in [9] but we will reproduce their RGEs here for convenience - we ignore all except the top yukawa coupling (we found that our two new yukawas do not have a significant effect).

$$
(4 \pi)^{2} \lambda_{t}=\lambda_{t}\left[-3 \sum_{i=1}^{3} 4 \pi c_{i} \alpha_{i}+\frac{9}{2} \lambda_{t}^{2}+\frac{1}{2}\left(\lambda_{u}^{2}+\lambda_{d}^{2}\right)\right]
$$

with $c=\left(\frac{17}{60}, \frac{3}{4}, \frac{8}{3}\right)$.

The two-loop coupled RGEs can be solved analytically if we approximate the top yukawa coupling as a constant over the entire range of integration (see [36] for a study on the validity of this approximation). The solution is

$$
\alpha_{i}^{-1}(M)=\alpha_{G}^{-1}+\frac{1}{2 \pi} b^{M M} \ln \frac{\Lambda}{M}+\frac{1}{4 \pi} \sum_{j=1}^{3} \frac{B_{i j}^{M M}}{b_{j}^{M M}} \ln \left(1+\frac{1}{4 \pi} b_{j}^{M M} \alpha_{G}(\Lambda) \ln \frac{\Lambda}{M}\right)-\frac{1}{32 \pi^{3}} d_{i} \lambda_{t}^{2} \ln \frac{\Lambda}{M}
$$

\section{References}

[1] S. Perlmutter et al. [Supernova Cosmology Project Collaboration], "Measurements of Omega and Lambda from 42 High-Redshift Supernovae," Astrophys. J. 517 (1999) 565, astro-ph/9812133 
[2] D. N. Spergel et al. [WMAP Collaboration], "First Year Wilkinson Microwave Anisotropy Probe (WMAP) Observations: Determination of Cosmological Parameters," Astrophys. J. Suppl. 148 (2003) 175, astro-ph/0302209 C. L. Bennett et al., "First Year Wilkinson Microwave Anisotropy Probe (WMAP) Observations: Preliminary Maps and Basic Results," Astrophys. J. Suppl. 148, 1 (2003), astro-ph/0302207.

[3] R. Barbieri and A. Strumia, "What is the limit on the Higgs mass?," Phys. Lett. B 462, 144 (1999), hep-ph/9905281.

[4] L. Giusti, A. Romanino and A. Strumia, "Natural ranges of supersymmetric signals," Nucl. Phys. B 550 (1999) 3, hep-ph/9811386.

[5] S. Weinberg, "Anthropic Bound On The Cosmological Constant," Phys. Rev. Lett. 59 (1987) 2607.

[6] V. Agrawal, S. M. Barr, J. F. Donoghue and D. Seckel, "The anthropic principle and the mass scale of the standard model," Phys. Rev. D 57, 5480 (1998), hep-ph/9707380.

[7] N. Arkani-Hamed and S. Dimopoulos, "Supersymmetric unification without low energy supersymmetry and signatures for fine-tuning at the LHC," JHEP 0506, 073 (2005) arXiv:hep-th/0405159.

[8] N. Arkani-Hamed, S. Dimopoulos and S. Kachru, "Predictive landscapes and new physics at a TeV," arXiv:hep-th/0501082

[9] G. F. Giudice and A. Romanino, "Split supersymmetry," Nucl. Phys. B 699, 65 (2004) [Erratumibid. B 706, 65 (2005)], hep-ph/0406088

[10] M. W. Goodman and E. Witten, "Detectability Of Certain Dark-Matter Candidates," Phys. Rev. D 31, 3059 (1985).

[11] D. S. Akerib et al. [CDMS Collaboration], "Limits on spin-independent WIMP nucleon interactions from the two-tower run of the Cryogenic Dark Matter Search," arXiv:astro-ph/0509259.

[12] D. R. Smith and N. Weiner, "Inelastic dark matter," Phys. Rev. D 64, 043502 (2001), hep-ph/0101138.

[13] A. Pierce, "Dark matter in the finely tuned minimal supersymmetric standard model," Phys. Rev. D 70 (2004) 075006, hep-ph/0406144.

[14] A. Masiero, S. Profumo and P. Ullio, "Neutralino dark matter detection in split supersymmetry scenarios," Nucl. Phys. B 712, 86 (2005) arXiv:hep-ph/0412058. L. Senatore, "How heavy can the fermions in split SUSY be? A study on gravitino and extradimensional LSP," Phys. Rev. D 71, 103510 (2005) arXiv:hep-ph/0412103.

[15] P. Gondolo, J. Edsjo, P. Ullio, L. Bergstrom, M. Schelke and E. A. Baltz, "DarkSUSY: A numerical package for supersymmetric dark matter calculations," astro-ph/0211238 J. Edsjo, M. Schelke, P. Ullio and P. Gondolo, "Accurate relic densities with neutralino, chargino and sfermion coannihilations in mSUGRA," JCAP 0304, 001 (2003), hep-ph/0301106; P. Gondolo and G. Gelmini, "Cosmic Abundances Of Stable Particles: Improved Analysis," Nucl. Phys. B 360, 145 (1991). 
[16] A. Provenza, M. Quiros and P. Ullio, "Electroweak baryogenesis, large Yukawas and dark matter," JHEP 0510, 048 (2005) arXiv:hep-ph/0507325.

[17] J. Edsjo and P. Gondolo, "Neutralino relic density including coannihilations," Phys. Rev. D 56, 1879 (1997), hep-ph/9704361;

[18] P. L. Brink et al. [CDMS-II Collaboration], "Beyond the CDMS-II dark matter search: SuperCDMS," eConf C041213, 2529 (2004) arXiv:astro-ph/0503583. Useful comparison can be done using the tools of http://dendera.berkeley.edu/plotter/entryform.html

[19] D. Chang, W. F. Chang and W. Y. Keung, "Electric dipole moment in the split supersymmetry models," Phys. Rev. D 71 (2005) 076006, hep-ph/0503055

[20] N. Arkani-Hamed, S. Dimopoulos, G. F. Giudice and A. Romanino, "Aspects of split supersymmetry," Nucl. Phys. B 709, 3 (2005) arXiv:hep-ph/0409232.

[21] P. Gondolo and K. Freese, "CP-violating effects in neutralino scattering and annihilation," JHEP 0207, 052 (2002), hep-ph/9908390

[22] T. Falk, A. Ferstl and K. A. Olive, "Variations of the neutralino elastic cross-section with CP violating phases," Astropart. Phys. 13, 301 (2000) arXiv:hep-ph/9908311.

[23] B. C. Regan, E. D. Commins, C. J. Schmidt and D. DeMille, "New limit on the electron electric dipole moment," Phys. Rev. Lett. 88 (2002) 071805.

[24] D. Kawall, F. Bay, S. Bickman, Y. Jiang and D. DeMille, "Progress towards measuring the electric dipole moment of the electron in metastable PbO," AIP Conf. Proc. 698 (2004) 192.

[25] S. K. Lamoreaux, "Solid state systems for electron electric dipole moment and other fundamental measurements," nucl-ex/0109014.

[26] Y. K. Semertzidis, "Electric dipole moments of fundamental particles," arXiv:hep-ex/0401016.

[27] S. Eidelman et al. [Particle Data Group], "Review of particle physics," Phys. Lett. B 592 (2004) 1.

[28] I. Dorsner and P. F. Perez, "How long could we live?," Phys. Lett. B 625, 88 (2005) arXiv:hep-ph/0410198.

[29] I. Antoniadis, A. Delgado, K. Benakli, M. Quiros and M. Tuckmantel, "Splitting extended supersymmetry," arXiv:hep-ph/0507192,

[30] Y. Kawamura, "Gauge symmetry reduction from the extra space $\mathrm{S}(1) / \mathrm{Z}(2)$," Prog. Theor. Phys. 103, 613 (2000), hep-ph/9902423 A. Hebecker and J. March-Russell, "A minimal S(1)/(Z(2) x Z'(2)) orbifold GUT," Nucl. Phys. B 613, 3 (2001), hep-ph/0106166. L. J. Hall and Y. Nomura, "Gauge coupling unification from unified theories in higher dimensions," Phys. Rev. D 65, 125012 (2002), hep-ph/0111068. G. Altarelli and F. Feruglio, "SU(5) grand unification in extra dimensions and proton decay," Phys. Lett. B 511, 257 (2001), hep-ph/0102301. L. J. Hall and Y. Nomura, "Grand unification in higher dimensions," Annals Phys. 306, 132 (2003), hep-ph/0212134. P. C. Schuster, "Grand unification in higher dimensions with split supersymmetry," hep-ph/0412263. A. B. Kobakhidze, "Proton stability in TeV-scale GUTs," Phys. Lett. B 514, 131 (2001) arXiv:hep-ph/0102323. 
[31] I. Antoniadis, C. Kounnas and K. Tamvakis, "Simple Treatment Of Threshold Effects," Phys. Lett. B 119, 377 (1982).

[32] R. Contino, L. Pilo, R. Rattazzi and E. Trincherini, "Running and matching from 5 to 4 dimensions," Nucl. Phys. B 622, 227 (2002), hep-ph/0108102.

[33] S. Weinberg, "Effective Gauge Theories," Phys. Lett. B 91, 51 (1980).

[34] L. J. Hall, "Grand Unification Of Effective Gauge Theories," Nucl. Phys. B 178, 75 (1981).

[35] Z. Chacko, M. A. Luty and E. Ponton, "Massive higher-dimensional gauge fields as messengers of supersymmetry breaking," JHEP 0007, 036 (2000), hep-ph/9909248.

[36] P. Langacker and N. Polonsky, "Uncertainties in coupling constant unification," Phys. Rev. D 47, 4028 (1993), hep-ph/9210235.

[37] L. J. Hall and Y. Nomura, "A complete theory of grand unification in five dimensions," Phys. Rev. D 66, 075004 (2002), hep-ph/0205067.

[38] A. Hebecker and J. March-Russell, "Proton decay signatures of orbifold GUTs," Phys. Lett. B 539, 119 (2002), hep-ph/0204037.

[39] Y. Hayato et al. [Super-Kamiokande Collaboration], "Search for proton decay through $\mathrm{p} \rightarrow$ antinu K+ in a large water Cherenkov detector," Phys. Rev. Lett. 83 (1999) 1529, hep-ex/9904020.

[40] Y. Suzuki et al. [TITAND Working Group], "Multi-Megaton water Cherenkov detector for a proton decay search: TITAND (former name: TITANIC)," hep-ex/0110005. 